\title{
Brainstem Glioma in Adults
}

\author{
Jethro $\mathrm{Hu}^{1,2 *}$, Stephen Western ${ }^{3}$ and Santosh Kesari ${ }^{4 *}$ \\ ${ }^{1}$ Department of Neurology, Cedars-Sinai Medical Center, Los Angeles, CA, USA, ²Department of Neurosurgery, Cedars- \\ Sinai Medical Center, Los Angeles, CA, USA, ${ }^{3}$ Independent Researcher, Vancouver, Canada, ${ }^{4}$ Department of Translational \\ Neuro-Oncology and Neurotherapeutics, John Wayne Cancer Institute, Pacific Neuroscience Institute, Providence Saint \\ John's Health Center, Santa Monica, CA, USA
}

OPEN ACCESS

Edited by:

David D. Eisenstat,

University of Alberta, Canada

Reviewed by:

Justin Lathia,

Cleveland Clinic Lerner College of

Medicine, USA

Arnab Chakravarti,

The Ohio State University, USA

Todd Graham Mainprize,

University of Toronto, Canada

Jeffrey Raizer,

Northwestern University, USA

*Correspondence:

Jethro Hu

jethro.hu@cshs.org;

Santosh Kesari

kesaris@jwci.org

Specialty section:

This article was submitted to

Neuro-Oncology,

a section of the journal

Frontiers in Oncology

Received: 06 February 2016

Accepted: 20 July 2016

Published: 09 August 2016

Citation:

Hu J, Western S and Kesari S (2016) Brainstem Glioma in Adults.

Front. Oncol. 6:180.

doi: 10.3389/fonc.2016.00180
Brainstem gliomas are not nearly as common in adults as they are in children. They are likely the final common consequence not of a single disease process but of several. They can be difficult to diagnose, and are challenging to treat. Clinical studies of this diagnosis are few and generally small. Because of these factors, our understanding of the biology of adult brainstem glioma is incomplete. However, the knowledge base is growing and progress is being made. In this article, we review the current state of knowledge for brainstem glioma in adults and identify key areas for which additional information is required.

Keywords: DIPG, glioma, IDH mutations

\section{INTRODUCTION}

The past several years have yielded important insights into the biology of glioma in adults. Efforts such as The Cancer Genome Atlas (TCGA) have comprehensively cataloged the litany of somatic alterations occurring in glioblastoma and lower grade gliomas, leading to discoveries such as the importance of $I D H 1$ mutations in the development of a low-grade glioma and secondary glioblastoma (1). Pediatric diffuse intrinsic pontine glioma (DIPG) - the most frequent malignant primary brain tumor of childhood - on the other hand, has been found to have substantially different biological underpinnings. Eighty percent of these tumors, for example, contain mutations in genes that encode histone $\mathrm{H} 3(2)$.

At the nexus of these two diagnoses is brainstem glioma in adults. Unfortunately, it is perhaps more apt to describe adult brainstem glioma as the valley shrouded in shadow between the two growing mountains of knowledge that represent adult glioma and pediatric DIPG, as few studies have investigated this particular diagnosis. The anatomical basis of adult brainstem glioma does not lend itself to easy study, as the brainstem is packed full of brainstem nuclei and white matter tracts that are essential for basic functions. Resection is often not possible; even biopsies are challenging with significant risk for complications.

Within the umbrella of adult brainstem glioma, there is a subset of tumors that is similar in appearance and potentially similar in etiology to childhood DIPG, but with slightly older onset, generally affecting young adults. There is another subset of more well-circumscribed lesions that shares clinical and radiographic features with pilocytic astrocytoma, also with a younger median age at onset. The subset of adult brainstem gliomas presenting in later adulthood are typically more akin to other de novo malignant gliomas of adulthood. Yet despite these differences, there are also commonalities between these entities in terms of clinical considerations.

In this article, we will review the current state of knowledge (and lack of knowledge) for adult brainstem glioma and highlight prospects for advances in therapy. 


\section{CLINICAL FEATURES}

\section{Appearance and Presentation}

In contrast to pediatric DIPG, which accounts for approximately $20 \%$ of pediatric primary brain neoplasms, adult brainstem glioma constitutes less than $2 \%$ of adult gliomas, with a slight male preponderance $(3,4)$. Median age at diagnosis is in the mid30 s, though brainstem gliomas can present in any decade of life.

As befits a diagnosis that encompasses an array of pathophysiologic alterations, the radiographic appearance of adult brainstem glioma varies widely, with approximately $40 \%$ demonstrating enhancement $(3,5)$. By comparison, in pediatric DIPG, contrast enhancement is usually not a prominent feature. Contrast enhancement itself can take on a variable appearance, with some tumors exhibiting minimal or partial enhancement, and others showing robust enhancement. MR spectroscopy can be a useful aid for diagnosis, as elevation of the choline/NAA ratio is often detectable, with one analysis of adult brainstem gliomas showing this finding in $100 \%$ of cases analyzed (3). FDG-PET may also be helpful in differentiating aggressive lesions from indolent ones (6).

Brainstem gliomas are centered in the pons in approximately $60 \%$ of cases, but can also arise from the midbrain or medulla, and can infiltrate beyond the brainstem (7). They can be exophytic or expansile on imaging, or, as is the case with classic DIPG, can be infiltrative and diffuse with little notable mass effect (Figure 1).

Clinically, it is important to distinguish between gliomas that involve the midbrain tectum - which often behave indolently - and classic diffuse pontine gliomas, which often do not enhance with contrast or have varying enhancement patterns. Tectal gliomas are typically low-grade, non-enhancing, and often non-progressive. They are diagnosed most frequently in childhood, but can be detected at any age. Observation is often a reasonable management option, and when intervention is warranted, often it is only to address obstructive hydrocephalus. A small subset of tectal glioma may behave more aggressively; contrast enhancement and the development of cystic changes may herald such progression (8). Because of their distinct natural history, tectal gliomas are generally not considered in the same vein as other brainstem gliomas, and will not be further addressed in this review.

Histologically, adult brainstem gliomas can have an astrocytic, oligodendroglial, or mixed appearance, with astrocytic tumors further characterized as either pilocytic or diffusely infiltrative. A single-institution Italian retrospective analysis of 21 patients with histologically confirmed disease identified 2 pilocytic astrocytomas, 9 low-grade astrocytomas, 8 anaplastic astrocytomas, and 1 glioblastoma (3). An MD Anderson retrospective analysis that included 98 cases with histology identified 28 glioblastomas, 43 anaplastic astrocytomas, 15 diffuse astrocytomas, and 11 gliomas not otherwise specified (5).

The most frequent presenting symptom for adult brainstem glioma is headache, which can be a manifestation of hydrocephalus. Cranial nerve deficits and long tract signs are also common. "Crossed" deficits, in which facial signs and symptoms are contralateral from arm/leg signs and symptom, are another characteristic hallmark of brainstem pathology. Occasionally, clinical decline can precede radiographic progression, as the density of critical structures in the brainstem leaves little margin for growth before deficits occur.

\section{Prognostic Considerations}

Whereas pediatric DIPG is associated with a dismal prognosis of 10 months, with only $10 \%$ of pediatric patients living $>2$ years beyond diagnosis, median survival for adult brainstem glioma is in the grim-but-not-quite-as-dismal range of 30-40 months (5). Furthermore, because adult brainstem gliomas vary widely in their aggressiveness, prognosticating outcome for an individual patient at diagnosis can be difficult.

Increasing tumor grade and contrast enhancement are associated with significantly reduced survival. An analysis of 17
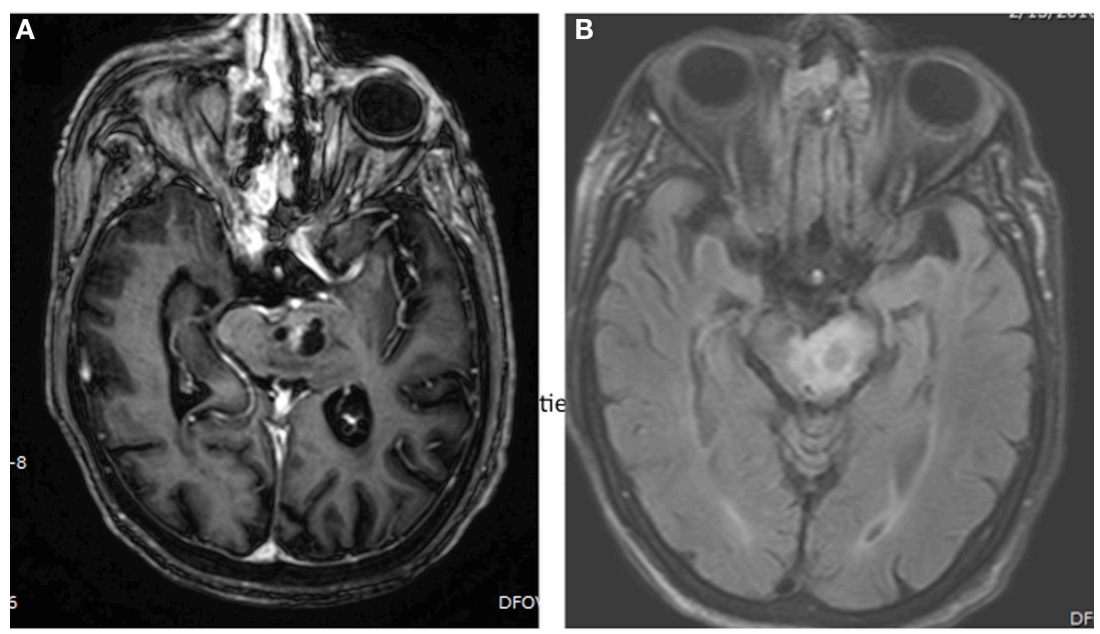

FIGURE 1 | Brain MRI showing presence of brainstem glioma in a 82-year-old-woman with histological diagnosed astrocytoma, grade III. (A) Axial, post-gadolinium MRI sequences showing a cystic, enhancing left pontine mass. (B) Axial, FLAIR MRI sequences showing surrounding edema in the left pontine mass. 
adults with DIPG demonstrated a median OS of 57 months for low-grade vs. 16 months for high-grade gliomas (9). Contrast enhancement correlated strongly with histological grade in this study - median OS in patients with non-enhancing DIPG was 57 vs. 13 months for patients with enhancing tumors. The MD Anderson retrospective analysis of adult brainstem glioma patients demonstrated a median OS of 77.0 months for WHO grade 2 diffuse astrocytoma, 21.1 months for WHO grade 3 anaplastic astrocytoma, and 14.8 months for glioblastoma (5).

Increasing age is also associated with worsened survival. The MD Anderson retrospective study demonstrated median survival of 34 months for patients 22-59 years of age, but only 14.2 months for patients 60 and over (5).

Other characteristics that have been identified as favorable prognostic factors include duration of symptoms $>3$ months, and the presence of "necrosis" on MRI $(9,10)$. Location also factors into prognosis - the MD Anderson study demonstrated a median survival of 66.0 months for midbrain tumors, 25.3 months for pontine tumors, and 51.3 months for medullary tumors (5). Predisposing factors are not well-defined, though there is one case report of a brainstem glioma developing 8 years after radiation treatment for a pituitary adenoma (11).

\section{Treatment Options}

For pediatric DIPG, standard of care treatment consists of involved field radiation therapy, typically to a dose of approximately 54-60 Gy. Alternative fractionation schemes for pediatric DIPG - including hyperfractionated, accelerated, and hypofractionated regimens - do not improve survival, with significantly increased risks of radiation toxicity at doses $>64 \mathrm{~Gy}$ (12).

To date, pediatric treatment trials for DIPG have failed to identify a significant benefit with chemotherapy. A phase 2 trial of the oral alkylating drug temozolomide along with radiation followed by adjuvant temozolomide - similar to the standard Stupp regimen for adult malignant glioma - resulted in median OS of only 9.5 months (13).

Other failed strategies in the pediatric DIPG population include high-dose chemotherapy, and metronomic approaches with etoposide, trophosphamide, or temozolomide (14-16). Several phase I and phase II trials of targeted therapy have been conducted, including trials of anti-EGFR drugs such as erlotinib, gefitinib, and nimotuzumab; trials targeting PDGFR using imatinib; trials utilizing the farnesyltransferase inhibitors tipifarnib and lonafarnib; trials of antiangiogenic drugs such as bevacizumab, semaxanib and the integrin inhibitor cilengitide; a trial of the mTOR inhibitor everolimus; and a trial of the multikinase inhibitor vandetanib (17-27). Overall, the results of these trials have been unimpressive, though long-term disease control has been reported in a small subset of these patients.

For adults with brainstem glioma, involved field radiation therapy, typically to a dose of 54-60 Gy, is considered standard upfront therapy, as is the case with pediatric DIPG. Few studies utilizing chemotherapy for the treatment of adult brainstem glioma have been performed, and the ones that have are often limited to single-arm, single-institution, or retrospective analyses. However, there is reason to hope that chemotherapeutic treatment for adults is more fruitful. The final results of RTOG
9802 demonstrate a clear benefit for combined chemoradiation vs. radiation therapy alone for patients with so-called "high-risk" grade 2 glioma, defined in this study as patients older than 40 or who had undergone subtotal resection. The benefits of combined treatment were particularly striking in patients with tumors that harbor an IDH1 mutation. Although the recently published results do not delineate how many of the 251 patients enrolled on this study had brainstem disease, the study adds to the body of evidence supporting a combined treatment approach for adult glioma patients of any age.

Perhaps the best line of evidence supporting the use of upfront chemotherapy (along with radiation) for adult brainstem glioma comes from the MD Anderson retrospective analysis, which demonstrated a striking difference in survival in adults with brainstem glioblastoma treated with the standard Stupp regimen (concurrent radiation and temozolomide followed by adjuvant temozolomide) vs. those who were not - median OS for patients treated with the Stupp regimen was 23.1 vs. 4.0 months for patients who were not (5). Of course, the scope of this analysis is limited by its small numbers (28 patients) and retrospective nature, as patients treated with the Stupp regimen tended to have better performance status at baseline (KPS 90 vs. 80), and were treated in the temozolomide era in which other advances in treatment and management may influence survival. A singleinstitution retrospective analysis of 15 adults with "low grade" diffuse brainstem glioma (defined either histologically or by radiographic appearance) treated with temozolomide at recurrence demonstrated a median PFS of 9.5 months and median OS of 14.4 months (9). Clinical improvement was noted in $60 \%$ of patients, and radiographic responses occurred in 6 of 15 patients (4 partial and 2 minor responses).

Re-irradiation is another potential treatment strategy for recurrent adult brainstem gliomas. A single-institution retrospective analysis of five adults with progressive/recurrent brainstem glioma treated with a repeat course of radiation resulted in post-treatment survival ranging from 3 to $36+$ months (28). Four of the five patients showed improvement in performance status post-treatment, with the other patient manifesting new symptoms potentially attributable to radiation toxicity.

The antiangiogenic drug bevacizumab may also have a role in the management of adults with brainstem glioma. A retrospective analysis that included 17 patients treated with bevacizumab at progression demonstrated median PFS of 2.0 months with 6 -month PFS of $21 \%$, including one patient who was progression free for 9 months (5). While somewhat encouraging, the 6-month PFS of $21 \%$ pales in comparison to the $40 \%$ 6-month PFS seen with supratentorial glioblastoma. It is also worth noting that bevacizumab may have a palliative benefit in addition to its anti-neoplastic activity. Bevacizumab reduces vasogenic cerebral edema, which frequently results in symptomatic improvement. Furthermore, this effect often allows patients to taper their steroid dose, which can have downstream benefits as well. Because bevacizumab both vasoconstricts and reduces vascular permeability, treatment with bevacizumab often mitigates contrast enhancement. Monitoring for changes on T2/FLAIR and DiffusionWeight Imaging (DWI) takes on additional importance in this circumstance. 


\section{SCIENTIFIC UNDERSTANDING}

Few scientific studies have specifically investigated brainstem glioma in adults, but our understanding of the biology of pediatric DIPG has been greatly enhanced over the past few years by several landmark studies (Table 1). In one such study published in 2012, Wu et al. identified mutations in H3F3A and HIST1H3B in $78 \%$ of pediatric DIPG (2). The mutations in both of these genes result in a $\mathrm{K} 27 \mathrm{M}$ amino acid substitution in histone $\mathrm{H} 3$, and suggest epigenetic dysregulation as an important contributor to the pathogenesis of pediatric DIPG. In contrast, histone H3 mutations rarely occur in adult supratentorial glioblastoma (29).

Somatic $I D H$ mutations, on the other hand, are very common in adult non-brainstem lower-grade (grade 2/3) gliomas. In the TCGA analysis of such tumors, $80.1 \%$ were found to harbor $I D H$ mutations. These mutations were previously thought to be uncommon in adult brainstem glioma, as studies of pediatric DIPG did not identify any such mutations, and IDH1 R132H mutations as detected by immunohistochemistry were found in only $6 \%$ of the adult brainstem gliomas in one study (5). However, a whole-exome sequencing study of brainstem glioma demonstrated that $I D H$ mutations are not altogether uncommon in the subset of tumors from adults, particularly when alternative non- $R 132 \mathrm{H}$ mutations (which constitute only $5 \%$ of $I D H$ mutations in adult supratentorial glioblastoma) are included (30). Of the 13 adult brainstem gliomas that underwent whole exome sequencing in this study, 5 (38\%) were IDH1 mutated. There were three IDH1 R132H mutations, one $R 132 \mathrm{C}$, and one $R 132 \mathrm{~L}$ mutation. All of these cases also had mutant TP53, and four of the cases had mutant ATRX. Genetically, this group resembles classic IDH1 mutant cerebral astrocytomas, which also usually harbor mutations in TP53 and ATRX. Mean age of these 5 patients was 40 and the predominant tumor location was in the pons, with all 5 having a pontine location, four of which were located both in the pons and medulla. Histopathology was oligoastrocytoma grade 2 or 3 in four cases and GBM in one. IDH1-mutant cases had a significantly better median survival than the IDH1 wildtype cases.

In the same study, H3F3A mutations were identified in $7 / 13$ (54\%) tumors. Each of these seven cases additionally harbored a mutation in either TP53 (four cases) or PPM1D (3 cases). PPM1D suppresses the activation of several DNA damage response mediators; the mutations identified in PPM1D enhanced this activity. One of these cases carried a mutation in PDGFRA (in addition to PPM1D). One had a mutation in NF1 (in addition to $P P M 1 D$ ). Mean age of these adult patients was 29, and the predominant location was in the medulla (four cases) and midbrain ( 2 cases). Two of the cases had dual location in the medulla and pons. Notably, while all the IDH1 mutant cases had a pontine location, only $2 / 7$ of the H3F3A mutant cases had a pontine location, and the $H 3 F 3 A$ mutant cases tended to be younger on average. Histopathology in this group was oligoastrocytoma (two cases), astrocytoma (two cases), oligodendroglioma (one case), and glioblastoma (two cases). All H3F3A mutations identified in this study involved the K27M amino acid substitution. No H3F3A G34 mutation or HIST1H3B mutations were detected. H3F3A mutations at G34 are typically found in the cerebral hemispheres and occur in adolescence and in young adults, in contrast with H3F3A mutations at K27, which are most common in brainstem gliomas of young children, but may also occur in adults as shown by this study $(9,29)$.

The frequency of MGMT promoter methylation in adult brainstem glioma has not been adequately evaluated, although positive MGMT expression (correlating with an unmethylated MGMT promoter) was found in $64.7 \%$ of cases in one series (31). $1 \mathrm{p} / 19 \mathrm{q}$ codeletion is rarely detected in adult brainstem glioma. A genomic profiling study of nine adult brainstem gliomas identified one BRAF V600E mutation and two PIK3CA mutations (5). Mutations in AVCR1 (encoding a type I activin A receptor kinase) are commonly seen in pediatric DIPG and may lead to overactivation of the BMP-TGF- $\beta$ signaling pathway $(32,33)$. However, the frequency of $A V C R 1$ mutations in adult brainstem glioma is unknown. Amplification of MYCN was found to define a subset of pediatric DIPG in one genomic analysis; the frequency of this finding in adult brainstem glioma is also unknown (34).

\section{FUTURE DIRECTIONS}

Brainstem gliomas, particularly in adults, are not one disease. Armed with genomic sequencing technology that is becoming

TABLE 1 | Summary of adult and pediatric brainstem gliomas.

\begin{tabular}{|c|c|c|c|}
\hline & Pediatric DIPG & Adult brainstem glioma & Adult supratentorial GBM \\
\hline Incidence & $20 \%$ of pediatric brain tumors & $2 \%$ of adult glioma & $80 \%$ of primary malignant brain tumors \\
\hline Median age of onset & $5-9$ & mid-30s & 64 \\
\hline MRI contrast enhancement & Usually nonenhancing & Variable enhancement & Strong enhancement \\
\hline Significant gene alterations & $\begin{array}{l}\text { HIST1H3B } \\
\text { H3F3A (K27M) } \\
\text { TP53 } \\
\text { AVCR1 } \\
\text { PPM1D } \\
\text { MYC } \\
\text { NTRK fusions }\end{array}$ & $\begin{array}{l}\text { IDH1 } \\
\text { H3F3A (K27M) }\end{array}$ & $\begin{array}{l}\text { MGMT promoter methylation } \\
\text { EGFR amplification } \\
\text { IDH1 and IDH2 } \\
\text { TERT promoter }\end{array}$ \\
\hline Standard initial treatment & $\mathrm{RT}$ & RT & RT and temozolomide \\
\hline Median overall survival & 10 months & 30-40 months (varies by grade) & $12-18$ months \\
\hline
\end{tabular}


increasingly sophisticated and widely available, the future of treatment for adult brainstem glioma lies in parsing out which tumors are most likely to respond to which therapies. Based on current knowledge, for example, it appears that most adult brainstem gliomas can be stratified into one of two groups based upon whether the tumor has an $I D H$ mutation or H3F3A mutation. Interestingly, $I D H$ mutations lead to DNA hypermethylation throughout the genome, while $H 3 F 3 A$ mutant tumors are associated with DNA hypomethylation $(30,35)$. So while both of these tumor types have a significant epigenetic basis, opposite strategies will be required to target the DNA methylation status of these two groups. Pharmacologic inhibition of histone H3 demethylation has shown promise in preclinical studies on H3F3A-mutant brainstem glioma cell lines, and small molecule H3 demethylase inhibitors are in development $(36,37)$. The multihistone deacetylase inhibitor panobinostat has also been shown to restore methylation and normalize gene expression in preclinical models of DIPG (38). Other studies suggest that somatic histone $\mathrm{H} 3$ alterations may result an increased expression of bromodomain-containing protein 1 and 4 (BRD1 and BRD4) (37). Tumors with these mutations may thus be susceptible to treatment with a bromodomain inhibitor, several of which are in development. The subset of brainstem gliomas with MYCN amplification may also be susceptible to bromodomain inhibition (39).

Brainstem gliomas that harbor IDH mutations, on the other hand, may respond to hypomethylating agents, such as 5-azacytidine and decitabine, both FDA approved for myelodysplastic syndrome. Though not yet confirmed by clinical trials, at least three preclinical studies have demonstrated a therapeutic effect of DNA hypomethylating agents on IDH1-mutant tumor cell lines (40-42). In one of these studies, 5-azacytidine was administered intraperitoneally to nude mice bearing subcutaneous xenografts derived from an IDH1-mutant anaplastic astrocytoma specimen. At week 14, tumor regression was observed in the 5-azacytidinetreated mice, in stark contrast with the control mice (41). A recent study outlined a novel mechanism involved in the pathogenesis of $I D H$-mutant gliomas: methylation of insulator protein binding sites within DNA, leading to aberrant contact of the PDGFRA promoter with distant enhancer elements, causing overexpression of PDGFRA, a known glioma oncogene (42). In vitro treatment of IDH1-mutant BT142 gliomaspheres with 5-azacytidine reduced methylation of the insulator protein binding site by $\sim 2.5$-fold and downregulated PDGFRA expression 5-fold. Phase I pharmacokinetic/pharmacodynamic trials would be needed to confirm the ability of DNA hypomethylating drugs such as 5-azacytidine and decitabine to reach tumors within the central nervous system at therapeutic concentrations.

A phase I trial of AG-120, an oral inhibitor of mutant IDH1, demonstrated stable disease in 10 of 20 patients with IDH1mutant glioma, with a 6-month clinical benefit response rate of $25 \%$. Further testing is ongoing. Other mutations that have been reported in adult brainstem glioma, such as mutations in PDGFRA, PIK3CA, and PPM1D, are also potentially targetable with drugs that are either already commercially available or in development.
Incorporating genomic data into a strategy for treatment - an approach called "precision medicine" by some - also faces significant challenges. For this strategy to get off the ground, obtaining tumor tissue for genomic analysis is a prerequisite. While operating on the brainstem requires great expertise and care, the precision with which a neurosurgeon can operate these days, coupled with decreasing tissue requirements for genomic analysis, makes obtaining tissue on a standard basis a much more reasonable option now.

Another challenge in adopting a precision medicine approach is figuring out how to incorporate such a strategy into the traditional clinical trial framework. Adopting such methodology, a clinical trial for pediatric patients with DIPG - still ongoing, but not recruiting - uses the result of EGFR and MGMT promoter methylation testing to determine whether patients receive temozolomide and/or erlotinib in addition to bevacizumab and irradiation (NCT01182350). Unfortunately, the incidence of adult brainstem glioma is likely too low for anything larger than a small-scale trial to be performed. However, adults with brainstem glioma may still be candidates for clinical trials as participants in so-called "basket" trials that stratify patients by molecular alteration regardless of tumor histology. For example, adults with recurrent brainstem glioma harboring a $B R A F$ V600E mutation may qualify for the recently initiated NCI-MATCH study, which includes an arm for patients with tumors with BRAF V600E mutation to receive a combination of dabrafenib and trametinib. Patients may also seek to have genomic profiling performed on their tumor tissue outside the framework of a clinical trial. In these instances, patients must understand that using such data to dictate management is an asof-yet unproven treatment strategy, promising though it may be.

Immunotherapy may also one day play a role in the treatment of brainstem glioma in adults. Vaccine trials for adult malignant glioma have been conducted for over a decade, but until recently, these trials usually required patients to undergo gross-total or near-gross total resection, which precluded patients with brainstem glioma from participating. Recently, however, some vaccine trials have begun allowing patients with significant residual disease to participate. Vaccines that are manufactured using autologous tumor lysate often require more tissue than is available for patients with brainstem glioma. However, trials that utilize tumor-associated peptides usually have much less restrictive tissue requirements.

Immune checkpoint inhibitors that block targets such as PD1, PDL1, and CTLA4 have recently shown impressive activity across a range of advanced malignancies. These therapies have not yet been evaluated for brainstem glioma, though interest is high.

\section{CONCLUSION}

Because adult brainstem glioma is a collection of clinical and histological entities, individual patient treatment depends on symptoms, performance status, age, histology, molecular pathology, and availability of new therapeutic studies. IDH and histone mutations are relatively recent discoveries and are only now being introduced into upcoming pediatric studies; 
retrospective analyses are underway in selected adult patient populations. Given the rapid advances in the field in molecular classification, epigenetics and cancer therapeutics, it is likely that we will make significant gains in this vein over the next decade, thereby enabling evidence-based management algorithms for both pediatric and adult DIPG as well as the larger group of brainstem tumors. For now, we judiciously biopsy/resect patients whom we deem safe to do so for the purpose of symptomatic control and molecular testing for prognosis as well as for enrolling into therapeutic studies which usually require tissue diagnosis and mutation status (e.g., IDH1/2 mutation, BRAF mutation, Histone 3 mutation). Radiation and temozolomide

\section{REFERENCES}

1. Brat DJ, Verhaak RG, Aldape KD, Yung WK, Salama SR, Cooper LA, et al. Comprehensive, integrative genomic analysis of diffuse lower-grade gliomas. N Engl J Med (2015) 372(26):2481-98. doi:10.1056/NEJMoa1402121

2. Wu G, Broniscer A, McEachron TA, Lu C, Paugh BS, Becksfort J, et al. Somatic histone $\mathrm{H} 3$ alterations in pediatric diffuse intrinsic pontine gliomas and non-brainstem glioblastomas. Nat Genet (2012) 44(3):251-3. doi:10.1038/ ng.1102

3. Salmaggi A, Fariselli L, Milanesi I, Lamperti E, Silvani A, Bizzi A, et al. Natural history and management of brainstem gliomas in adults. A retrospective Italian study. J Neurol (2008) 255(2):171-7. doi:10.1007/s00415-008-0589-0

4. Reyes-Botero G, Mokhtari K, Martin-Duverneuil N, Delattre JY, LaigleDonadey F. Adult brainstem gliomas. Oncologist (2012) 17(3):388-97. doi:10.1634/theoncologist.2011-0335

5. Theeler BJ, Ellezam B, Melguizo-Gavilanes I, de Groot JF, Mahajan A, Aldape KD, et al. Adult brainstem gliomas: correlation of clinical and molecular features. JNeurol Sci (2015) 353(1-2):92-7. doi:10.1016/j. jns.2015.04.014

6. Treglia G, Muoio B, Del Ciello A, Bertagna F. Usefulness of (18)F-FDG-PET/ CT in evaluating a brainstem glioma in an adult patient with neurofibromatosis type 1. Nucl Med Mol Imaging (2013) 47(3):212-3. doi:10.1007/ s13139-013-0193-9

7. Kesari S, Kim RS, Markos V, Drappatz J, Wen PY, Pruitt AA. Prognostic factors in adult brainstem gliomas: a multicenter, retrospective analysis of 101 cases. J Neurooncol (2008) 88(2):175-83. doi:10.1007/s11060-008-9545-1

8. Guillamo JS, Monjour A, Taillandier L, Devaux B, Varlet P, Haie-Meder C, et al. Brainstem gliomas in adults: prognostic factors and classification. Brain (2001) 124(Pt 12):2528-39. doi:10.1093/brain/124.12.2528

9. Reyes-Botero G, Laigle-Donadey F, Mokhtari K, Martin-Duverneuil N, Delattre JY. Temozolomide after radiotherapy in recurrent "low grade" diffuse brainstem glioma in adults. J Neurooncol (2014) 120(3):581-6. doi:10.1007/ s11060-014-1589-9

10. Ueoka DI, Nogueira J, Campos JC, Maranhão Filho P, Ferman S, Lima MA. Brainstem gliomas - retrospective analysis of 86 patients. J Neurol Sci (2009) 281(1-2):20-3. doi:10.1016/j.jns.2009.03.009

11. Abboud SE, Wolansky LJ, Manjila SV, Lo SS, Arafah BM, Selman WR, et al. Histologically proven radiation-induced brainstem glioma 93 months after external beam radiotherapy for pituitary macroadenoma: radiation treatment dose and volume correlation. J Neuroimaging (2015) 25(4):674-6. doi:10.1111/ jon.12181

12. Bartels U, Hawkins C, Vézina G, Kun L, Souweidane M, Bouffet E. Proceedings of the diffuse intrinsic pontine glioma (DIPG) Toronto Think Tank: advancing basic and translational research and cooperation in DIPG. J Neurooncol (2011) 105(1):119-25. doi:10.1007/s11060-011-0704-4

13. Bailey S, Howman A, Wheatley K, Wherton D, Boota N, Pizer B, et al. Diffuse intrinsic pontine glioma treated with prolonged temozolomide and radiotherapy - results of a United Kingdom phase II trial (CNS 2007 04). Eur J Cancer (2013) 49(18):3856-62. doi:10.1016/j.ejca.2013.08.006

14. Wolff JE, Westphal S, Mölenkamp G, Gnekow A, Warmuth-Metz M, Rating D, et al. Treatment of paediatric pontine glioma with oral trophosphamide and etoposide. Br J Cancer (2002) 87(9):945-9. doi:10.1038/sj.bjc.6600552 are used routinely upfront and re-radiation is often offered at recurrence. We still have much to learn about the pathogenesis of adult brainstem glioma, but landmark studies on pediatric DIPG on one side and adult non-brainstem glioma on the other is likely to have a considerable degree of relevance. As our understanding of adult brainstem glioma increases, better treatments are sure to follow.

\section{AUTHOR CONTRIBUTIONS}

$\mathrm{JH}, \mathrm{SW}$, and SK contributed to initial and subsequent drafts of the manuscript.

15. Sharp JR, Bouffet E, Stempak D, Gammon J, Stephens D, Johnston DL, et al. A multi-centre Canadian pilot study of metronomic temozolomide combined with radiotherapy for newly diagnosed paediatric brainstem glioma. Eur J Cancer (2010) 46(18):3271-9. doi:10.1016/j.ejca.2010.06.115

16. Korones DN, Fisher PG, Kretschmar C, Zhou T, Chen Z, Kepner J, et al. Treatment of children with diffuse intrinsic brain stem glioma with radiotherapy, vincristine and oral VP-16: a Children's Oncology Group phase II study. Pediatr Blood Cancer (2008) 50(2):227-30. doi:10.1002/pbc.21154

17. Geoerger B, Hargrave D, Thomas F, Ndiaye A, Frappaz D, Andreiuolo F, et al. Innovative therapies for children with cancer pediatric phase I study of erlotinib in brainstem glioma and relapsing/refractory brain tumors. Neuro Oncol (2011) 13(1):109-18. doi:10.1093/neuonc/noq141

18. Daw NC, Furman WL, Stewart CF, Iacono LC, Krailo M, Bernstein ML, et al. Phase I and pharmacokinetic study of gefitinib in children with refractory solid tumors: a Children's Oncology Group study. JClin Oncol (2005) 23(25):6172-80. doi:10.1200/JCO.2005.11.429

19. Massimino M, Bode U, Biassoni V, Fleischhack G. Nimotuzumab for pediatric diffuse intrinsic pontine gliomas. Expert Opin Biol Ther (2011) 11(2):247-56. doi:10.1517/14712598.2011.546341

20. Pollack IF, Jakacki RI, Blaney SM, Hancock ML, Kieran MW, Phillips P, et al. Phase I trial of imatinib in children with newly diagnosed brainstem and recurrent malignant gliomas: a Pediatric Brain Tumor Consortium report. Neuro Oncol (2007) 9(2):145-60. doi:10.1215/15228517-2006-031

21. Fouladi M, Nicholson HS, Zhou T, Laningham F, Helton KJ, Holmes E, et al. A phase II study of the farnesyl transferase inhibitor, tipifarnib, in children with recurrent or progressive high-grade glioma, medulloblastoma/primitive neuroectodermal tumor, or brainstem glioma: a Children's Oncology Group study. Cancer (2007) 110(11):2535-41. doi:10.1002/cncr.23078

22. Kieran MW, Packer RJ, Onar A, Blaney SM, Phillips P, Pollack IF, et al. Phase I and pharmacokinetic study of the oral farnesyltransferase inhibitor lonafarnib administered twice daily to pediatric patients with advanced central nervous system tumors using a modified continuous reassessment method: a Pediatric Brain Tumor Consortium study. J Clin Oncol (2007) 25(21):3137-43. doi:10.1200/JCO.2006.09.4243

23. Gururangan S, Chi SN, Young Poussaint T, Onar-Thomas A, Gilbertson RJ, Vajapeyam S, et al. Lack of efficacy of bevacizumab plus irinotecan in children with recurrent malignant glioma and diffuse brainstem glioma: a Pediatric Brain Tumor Consortium study. JClin Oncol (2010) 28(18):3069-75. doi:10.1200/JCO.2009.26.8789

24. Kieran MW, Supko JG, Wallace D, Fruscio R, Poussaint TY, Phillips P, et al. Phase I study of SU5416, a small molecule inhibitor of the vascular endothelial growth factor receptor (VEGFR) in refractory pediatric central nervous system tumors. Pediatr Blood Cancer (2009) 52(2):169-76. doi:10.1002/pbc.21873

25. MacDonald TJ, Stewart CF, Kocak M, Goldman S, Ellenbogen RG, Phillips $\mathrm{P}$, et al. Phase I clinical trial of cilengitide in children with refractory brain tumors: Pediatric Brain Tumor Consortium study PBTC-012. J Clin Oncol (2008) 26(6):919-24. doi:10.1200/JCO.2007.14.1812

26. Fouladi M, Laningham F, Wu J, O'Shaughnessy MA, Molina K, Broniscer A, et al. Phase I study of everolimus in pediatric patients with refractory solid tumors. J Clin Oncol (2007) 25(30):4806-12. doi:10.1200/JCO.2007.11.4017

27. Broniscer A, Baker JN, Tagen M, Onar-Thomas A, Gilbertson RJ, Davidoff $\mathrm{AM}$, et al. Phase I study of vandetanib during and after radiotherapy in children 
with diffuse intrinsic pontine glioma. JClin Oncol (2010) 28(31):4762-8. doi:10.1200/JCO.2010.30.3545

28. Susheela SP, Revannasiddaiah S, Muzumder S, Mallarajapatna G, Kallur $\mathrm{K}$, Basavalingaiah AS. Re-irradiation with hypo-fractionated stereotactic robotic radiotherapy for salvage in adult patients with brainstem glioma. Ecancermedicalscience (2013) 7:366. doi:10.3332/ecancer.2013.366

29. Schwartzentruber J, Korshunov A, Liu XY, Jones DT, Pfaff E, Jacob K, et al. Driver mutations in histone $\mathrm{H} 3.3$ and chromatin remodelling genes in paediatric glioblastoma. Nature (2012) 482(7384):226-31. doi:10.1038/nature10833

30. Zhang L, Chen LH, Wan H, Yang R, Wang Z, Feng J, et al. Exome sequencing identifies somatic gain-of-function PPM1D mutations in brainstem gliomas. Nat Genet (2014) 46(7):726-30. doi:10.1038/ng.2995

31. Babu R, Kranz PG, Agarwal V, McLendon RE, Thomas S, Friedman AH, et al. Malignant brainstem gliomas in adults: clinicopathological characteristics and prognostic factors. J Neurooncol (2014) 119(1):177-85. doi:10.1007/ s11060-014-1471-9

32. Buczkowicz P, Hoeman C, Rakopoulos P, Pajovic S, Letourneau L, Dzamba $\mathrm{M}$, et al. Genomic analysis of diffuse intrinsic pontine gliomas identifies three molecular subgroups and recurrent activating ACVR1 mutations. Nat Genet (2014) 46(5):451-6. doi:10.1038/ng.2936

33. Taylor KR, Mackay A, Truffaux N, Butterfield YS, Morozova O, Philippe C, et al. Recurrent activating ACVR1 mutations in diffuse intrinsic pontine glioma. Nat Genet (2014) 46(5):457-61. doi:10.1038/ng.2925

34. Wu G, Diaz AK, Paugh BS, Rankin SL, Ju B, Li Y, et al. The genomic landscape of diffuse intrinsic pontine glioma and pediatric non-brainstem high-grade glioma. Nat Genet (2014) 46(5):444-50. doi:10.1038/ng.2938

35. Turcan S, Rohle D, Goenka A, Walsh LA, Fang F, Yilmaz E, et al. IDH1 mutation is sufficient to establish the glioma hypermethylator phenotype. Nature (2012) 483(7390):479-83. doi:10.1038/nature10866

36. Hashizume R, Andor N, Ihara Y, Lerner R, Gan H, Chen X, et al. Pharmacologic inhibition of histone demethylation as a therapy for pediatric brainstem glioma. Nat Med (2014) 20(12):1394-6. doi:10.1038/nm.3716
37. Ramaswamy V, Remke M, Taylor MD. An epigenetic therapy for diffuse intrinsic pontine gliomas. Nat Med (2014) 20(12):1378-9. doi:10.1038/ nm.3769

38. Grasso CS, Tang Y, Truffaux N, Berlow NE, Liu L, Debily MA, et al. Functionally defined therapeutic targets in diffuse intrinsic pontine glioma. Nat Med (2015) 21(7):827. doi:10.1038/nm0715-827a

39. Puissant A, Frumm SM, Alexe G, Bassil CF, Qi J, Chanthery YH, et al. Targeting MYCN in neuroblastoma by BET bromodomain inhibition. Cancer Discov (2013) 3(3):308-23. doi:10.1158/2159-8290.CD-12-0418

40. Turcan S, Fabius AW, Borodovsky A, Pedraza A, Brennan C, Huse J, et al. Efficient induction of differentiation and growth inhibition in IDH1 mutant glioma cells by the DNMT inhibitor decitabine. Oncotarget (2013) 4(10):1729-36. doi:10.18632/oncotarget.1412

41. Borodovsky A, Salmasi V, Turcan S, Fabius AW, Baia GS, Eberhart CG, et al. 5 -azacytidine reduces methylation, promotes differentiation and induces tumor regression in a patient-derived IDH1 mutant glioma xenograft. Oncotarget (2013) 4(10):1737-47. doi:10.18632/oncotarget.1408

42. Flavahan WA, Drier Y, Liau BB, Gillespie SM, Venteicher AS, StemmerRachamimov AO, et al. Insulator dysfunction and oncogene activation in IDH mutant gliomas. Nature (2016) 529(7584):110-4. doi:10.1038/ nature16490

Conflict of Interest Statement: The authors declare that the research was conducted in the absence of any commercial or financial relationships that could be construed as a potential conflict of interest.

Copyright $\odot 2016 \mathrm{Hu}$, Western and Kesari. This is an open-access article distributed under the terms of the Creative Commons Attribution License (CC BY). The use, distribution or reproduction in other forums is permitted, provided the original author(s) or licensor are credited and that the original publication in this journal is cited, in accordance with accepted academic practice. No use, distribution or reproduction is permitted which does not comply with these terms. 